\title{
Extension of "Interfacial Adsorption Denaturation" Behavior Interpretation Based on Gibbs Monolayer Formation by Biomolecules
}

\author{
Yusuke Kimura', Yuki Mashiyama², Haruka Maruyama1, and Atsuhiro Fujimori ${ }^{1 *}$ \\ ${ }^{1}$ Graduate School of Science and Engineering, Saitama University, 255 Shimo-okubo, Sakura-ku, Saitama 338-8570, JAPAN \\ ${ }^{2}$ Faculty of Engineering, Saitama University, 255 Shimo-okubo, Sakura-ku, Saitama 338-8570, JAPAN
}

\begin{abstract}
Using glucose oxidase and salmon testis-derived DNA molecules, we sought to extend the recently proposed idea of interfacial adsorption denaturation. The surface pressure-time $(\pi-t)$ isotherm of the glucose oxidase Gibbs monolayer exhibited a rapid increase in surface pressure and a relatively prompt transition to a liquid condensed film. The appearance of this rapid liquid expansion phase occurred much earlier than that previously identified for lysozyme, trypsin, cytochrome $\mathbf{C}$, and luciferase. This experimental finding was linked to the number of hydrophobic residues in the constituent unit, and the number of hydrophobic residues in glucose oxidase was the highest among these biomolecules. On the other hand, DNA molecules do not have such hydrophobic groups, or present a positive surface on the $\pi$-t curve. However, interfacial adsorption occurred, and the existence of molecules at the air/water interface was confirmed, even in the two-dimensional gas phase state. Furthermore, it was confirmed that an increase in surface pressure was detected during the formation of a mixed film of DNA molecules and biomolecules, forming a stable Gibbs monolayer. This mimic the behavior of mixed monolayer formation with matrix molecules in Langmuir monolayers. Moreover, it was clarified that the interfacial adsorption denaturation behavior changed when pH dependence was evaluated considering the isoelectric point of the biomolecular group.
\end{abstract}

Key words: biomolecules, adsorption at air/water interface, Gibbs monolayer, transferred films, interfacial adsorption denaturation

\section{Introduction}

The raging Covid-19/SARS-CoV-2 pandemic threatens humanity ${ }^{1,2)}$. The world is under pressure to overcome this situation by acquiring collective immunity ${ }^{3)}$, or by developing vaccines ${ }^{4}$. It is viral mutations ${ }^{5}$ that should be feared, and it is extremely difficult to deal with viruses whose characteristics continually change, depending on the environment. The phenomenon of mutation and degeneration of biomolecules ${ }^{6,7)}$ often delivers a negative impression. It is easy to imagine the ineffectiveness of drugs against malignant bacteria ${ }^{8)}$ and the inactivation/loss of biomolecular functions ${ }^{9}$. However, on the other hand, there is an example in which heat-denatured lysozyme derived from egg white inactivated norovirus ${ }^{10)}$, and controlling the denaturation of biomolecules leads to the discovery of new biofunctions. From this point of view, conformational transition of biomolecules without deactivating inherent functionality has been studied, and is termed "interfacial ad- sorption denaturation" ${ }^{11,12)}$

Among the monolayers formed at the air/water interface, the Langmuir monolayer ${ }^{13-15}$ is a quasi-equilibrium state formed by external compression. On the other hand, the Gibbs monolayer ${ }^{16)}$ is formed in the equilibrium state between micelles and the interfacial adsorption film due to the presence of surfactant molecules in the aqueous solution (Fig. 1). In general, the former is described by the surface pressure-area $\left(\pi\right.$-A) isotherm ${ }^{17)}$, and the latter by the surface pressure-time $(\pi$-t $)$ isotherm ${ }^{18)}$. Langmuir films are water-insoluble monolayers ${ }^{19)}$ spread from an organic solvent. On the other hand, Gibbs monolayers are formed by water-soluble surfactant molecules ${ }^{20)}$. Biomolecules such as enzymes and proteins have abundant hydrophobic units, even though they are water-soluble in general, and their aqueous solution causes molecular adsorption at the air/water interface over time ${ }^{21)}$. During this time, it is thought that a conformational transition from a hydrophilic

*Correspondence to: Atsuhiro Fujimori, Graduate School of Science and Engineering, Saitama University, 255 Shimo-okubo, Sakura-ku, Saitama 338-8570, JAPAN

E-mail: fujimori@apc.saitama-u.ac.jp

Accepted November 26, 2020 (received for review August 11, 2020)

Journal of Oleo Science ISSN 1345-8957 print / ISSN 1347-3352 online

http://www.jstage.jst.go.jp/browse/jos/ http://mc.manusriptcentral.com/jjocs 


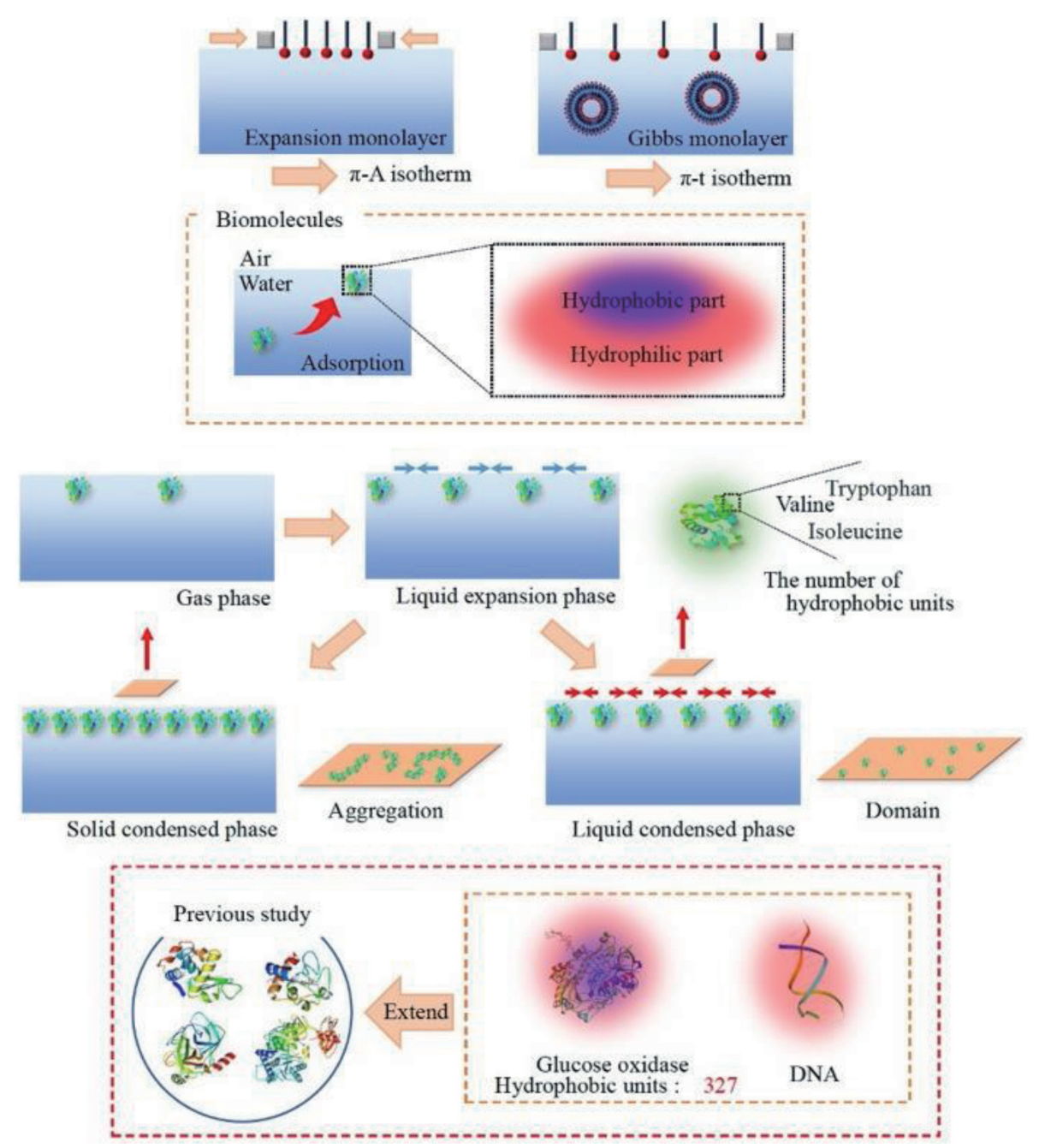

Fig. 1 Schematic illustrations of background and research strategy in this study.

conformation to an amphiphilic one occurs, and it has recently been argued that this conformational transition corresponds to "interfacial adsorption denaturation" ${ }^{11,12)}$. In this case, the concept of phase transition for Langmuir films has been applied to this phenomenon. That is to say, it corresponds to a two-dimensional gas phase ${ }^{22}$ if the distance between molecules at the interface is large, and the surface pressure is not detected. The stage where the surface pressure gradually rises corresponds to the liquid expansion phase ${ }^{23)}$, and interactions between the molecules are still weak. Subsequently, by means of two-dimensional phase transition, or alternatively a liquid condensation phase $^{24)}$, the solid condensed phase is achieved ${ }^{25)}$. The state in which the molecules are as densely packed as in the condensed phase indicates deactivation of the activi$\mathrm{ty}^{26)}$, which is not preferable for the functional utilization of biomolecular groups. On the other hand, although biomolecules in the two-dimensional expansion phase undergo low-affinity interactions in the lateral direction, these forces do not reach long-range order ${ }^{27}$. When utilizing in- terfacial adsorption denaturation, it is expected that the expansion phase indicates the potential ability. In other words, it is expected that the function will be enhanced and that the new function will arise along with the molecular conformation transition.

In addition, considering the stability of each phase ${ }^{28)}$, if the progress of interfacial adsorption denaturation is slow, and detection of the surface pressure/liquid expansion phase transition from the gas phase is difficult, the stability of the water-soluble molecular conformation is considered to be high. On the other hand, when the phase transition to the condensed state is rapid, it may be judged that it is an excellent material with high crystallinity as a monolayer substance, although it lacks stability as a biomolecule. In this case, abundant hydrophobic residues such as valine and isoleucine ${ }^{29,30)}$ are expected to be contained in the molecular unit, and the hydrophobic chain may be easily transferred to the air-side conformation at the air/water interface. In addition, the Gibbs monolayers of each biomolecule that transition to the solid condensed phase and the 
(a) Glucose oxidase Hydrophobic residues: 327

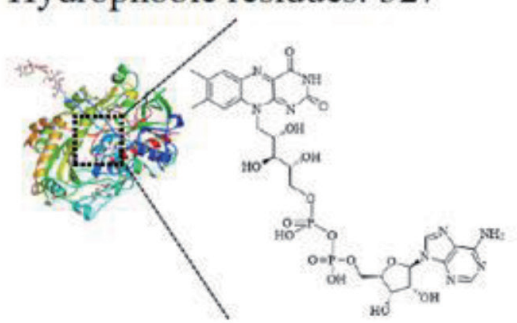

(c) Lysozyme

Hydrophobic residues: 57

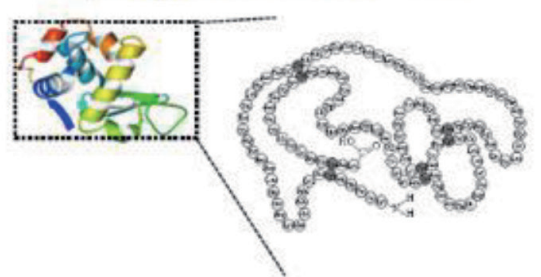

(e) Trypsin

Hydrophobic residues: 118

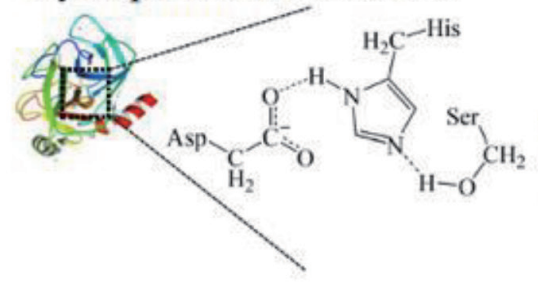

(b) DNA

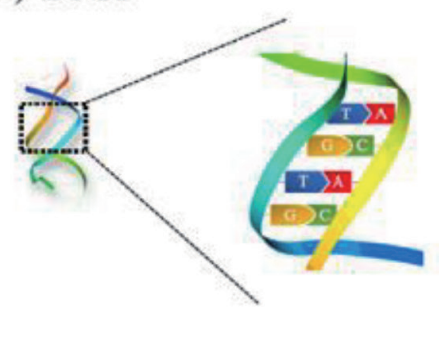

(d) Cytochrome C Hydrophobic residues: 46

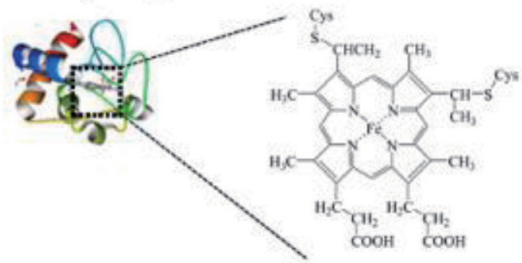

(f) Luciferase Hydrophobic residues: 284

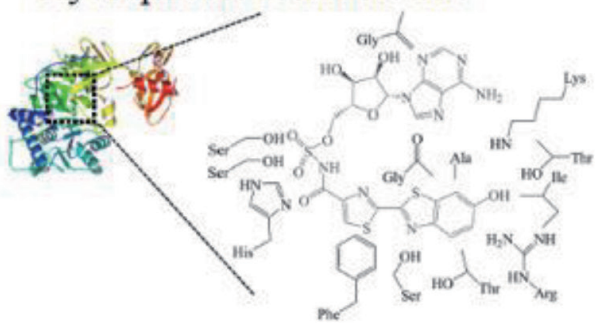

Fig. 2 Materials and experimental methods in this study.

liquid condensed phase tend to have different surface morphologies ${ }^{11,12)}$. At first, the domains are united with each other, and later, the domain size itself changes. A solid condensed phase is created by domains coalescing and forming a highly crystalline surface morphology.

In this research, we aimed to extend our theory as proposed above. First, we applied glucose oxidase ${ }^{31)}$, which is rich in hydrophobic units, and salmon testis-derived $\mathrm{DNA}^{32)}$, which has almost no hydrophobic units, to test this theory. These were evaluated as Gibbs monolayers formed from subphases, and then transferred onto a solid substrate to study their morphology and spectroscopic behavior. In addition, from changes in stability ${ }^{33)}$ before and after the biomolecular isoelectric point ${ }^{34)}$, the range of application of the theory was expanded. It is extremely important information that concentration and $\mathrm{pH}$ also affect dynamic surface tension and interfacial denaturation.

\section{Experimental Procedures}

2.1 Biomolecules as Gibbs monolayer-forming materials

The biomolecules mainly used in this study were glucose oxidase (Fig. 2(a)) and DNA molecules obtained from salmon sperm (Fig. 2(b)). These materials were purchased from Wako Pure Chemical Industries Ltd. Lysozyme enzyme(Fig. 2(c)), cytochrome C protein (Fig. 2(d)), trypsin digestive enzyme (Fig. $2(\mathrm{e})$ ), and luciferase luminescent enzyme(Fig. 2(f)) were also used. Egg white-derived lysozyme was purchased from Wako Pure Chemical Industries Ltd., with a molecular weight of approximately 14,000 Da. Cytochrome C protein and trypsin were derived from horse heart muscle and bovine pancreas, respectively, and were also purchased from the previously mentioned supplier. Luciferase enzyme was obtained from a bioluminescence kit purchased from Leave a Nest Co. Ltd. Stock solutions $\left(10 \mathrm{mg} \cdot \mathrm{L}^{-1}\right)$ of these biomolecules were prepared in $1 \mathrm{mM}$ phosphate buffer at $\mathrm{pH} 7$ (potassium and disodium hydrogenphosphates). 


\section{Y. Kimura, Y. Mashiyama, H. Maruyama et al.}

\subsection{Formation of Gibbs monolayers on the water surface and estimation of organized biomolecular films on solids}

In order to prepare the Gibbs monolayers, phosphate buffers containing the biomolecules were placed in a LB trough (USI Instruments, USI-3-22 Teflon-coated LB trough), and surface pressure-time $(\pi-\mathrm{t})$ isotherms were measured. In Gibbs monolayer experiments with biomolecules, it had been previously determined for the surface pressure to be zero after introducing the corresponding subphase aqueous solution into the LB trough. The adsorption behavior of biomolecules should also be related to the molar concentration of adsorbate molecules in the subphase water. Hence, the molar concentration of each biomolecule was kept constant at $10^{-6} \mathrm{M}$. This concentration is extremely dilute, and is in line with conditions previously reported $^{11,12)}$. The surface pressure-area $(\pi-\mathrm{A})$ isotherms were recorded at $2.4 \times 10^{-4} \% \cdot \mathrm{s}^{-1}$ compression speed. The temperature of the air/water interface was maintained at $15^{\circ} \mathrm{C}$ by circulating thermostatically controlled water around the trough. Measurements of the monolayer properties and LB film transfer were also performed using the LB trough. In this study, the formation of multilayers was carried out mainly by the horizontal lifting method ${ }^{35)}$. In this case, hydrophilic functional groups were forcibly exposed on the surface. Compared with the LB method, it is thought that there was a feature that was less likely to induce structural change because the process across the water surface is unnecessary. The surface morphologies of the transferred films were observed using a scanning probe microscope (SII, SPA300 with an SPI-3800 probe station) and microfabricated rectangular Si cantilevers. A cantilever spring constant of $1.4 \mathrm{~N} \cdot \mathrm{m}^{-1}$ was applied in this process. For atomic force microscopic (AFM) observation, the monolayers were transferred directly onto a newly cleaved mica substrate. AFM measurements were performed in dynamic force mode (DFM, tapping mode) to avoid damage to soft specimens. AFM measurements were also carried out in an air atmosphere to evaluate the maintenance of the three-dimensional structure of biomolecules adsorbed onto the solid substrate. In addition, for IR measurement, multilayers were fabricated onto a $\mathrm{CaF}_{2}$ substrate, the surface of which had been rendered hydrophobic with three LB layers of cadmium stearate.

\section{Results and Discussion}

\subsection{Investigation of interfacial adsorption denaturation of glucose oxidase using Gibbs monolayers}

Figure $3(\mathrm{a})$ shows the $\pi$-t isotherm $\left(15^{\circ} \mathrm{C}\right)$ of the Gibbs monolayer on the water surface of glucose oxidase. Compared with the behavior of biomolecules studied under the same conditions as depicted in Fig. $3(\text { b })^{11,12)}$, the surface pressure of the glucose oxidase Gibbs monolayer rose sharply. Immediately after that, the abrupt increase in surface pressure moderated, and the slope changed, suggesting a two-dimensional transition, as shown. After a period of 3 to $4 \mathrm{~h}$, a gradual transition was confirmed again, and a two-stage transition to the liquid expansion phase is indicated. The enlarged part in Fig. 3(b) is the initial slope of the $\pi$-t isotherm of each biomolecule. In other words, it indicates the part where the two-dimensional gas phase becomes a liquid expansion phase, and then the surface pressure decreases and monotonically increases. Lysozyme exhibited the longest two-dimensional gas phase with zero surface pressure, whereas the behavior of glucose oxidase was the opposite. It was confirmed that cytochrome $\mathrm{C}$ has a two-dimensional gas phase, but trypsin and luciferase have almost no such phase, and the surface pressure sharply increased. Although the initial gradient of trypsin showed a steep rise, luciferase presented a much higher surface pressure, suggesting the formation of a dense Gibbs monolayer. The rising behavior in the surface pressure of the glucose oxidase Gibbs monolayer overwhelms others, but the saturation surface pressure value did not only reach luciferase, but also trypsin and cytochrome C. In this study, these biomolecules adsorbed at the air/water interface are considered based on the conformation changes between the hydrophobic part and the hydrophilic parts. As a basis for this consideration, there are reports on the conformational changes of hydrophilic-hydrophobic groups during the formation of glucose oxidase-adsorbed monolayers ${ }^{36,37)}$. This study supports the results of these related papers.

Figure 3(c) schematically illustrates the transition from the gas phase to the liquid expansion phase, which corresponds to the detection of surface pressure. After that, glucose oxidase indicated the most rapid behavior, in which the surface pressure rose sharply compared to other biomolecules. In other words, the transition from the hydrophilic molecular conformation to the amphiphilic conformation, in which the hydrophobic group is exposed, causes adsorption at the air/water interface. At that time, it seems that the surface pressure can be detected as the two-dimensional interactions between the hydrophobic chains increase. This phase is considered to correspond to the liquid expansion phase expressed in the insoluble Langmuir monolayer. The steep rise in surface pressure seems to be closely related to the efficiency of transition to the amphipathic conformation.

In Fig. 4(a), the Gibbs monolayer behaviors of a total of five kinds of biomolecules, including four enzyme molecules, compared with glucose oxidase are summarized. With lysozyme, the longest period of $3.5 \mathrm{~h}$ was required to detect surface pressure. The surface pressure of cytochrome $\mathrm{C}$ was detected after $0.5 \mathrm{~h}$, and this time can be regarded as the transition from the two-dimensional gas 


\section{(a) Glucose Oxidase}

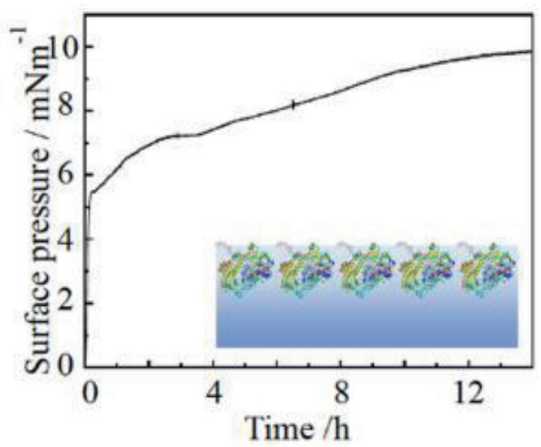

(b)

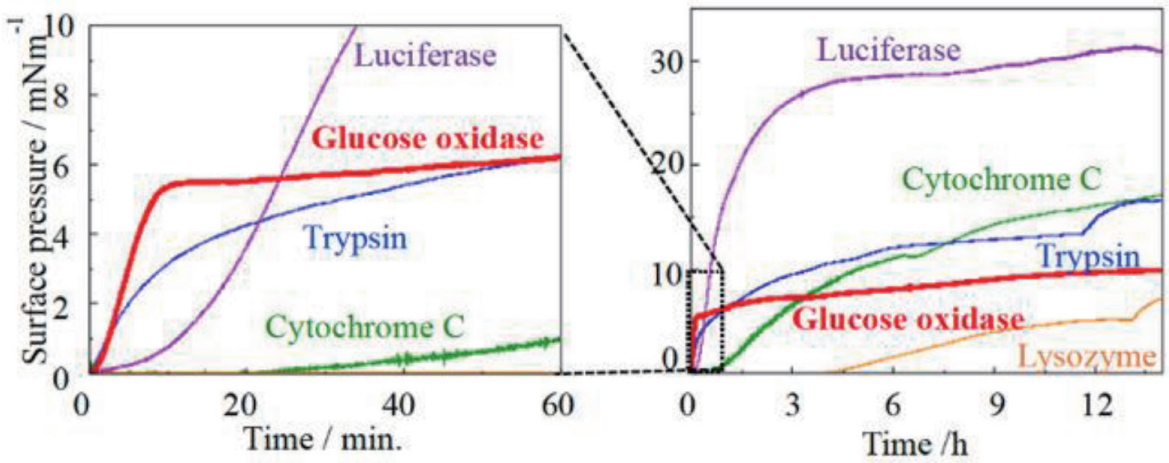

(c)

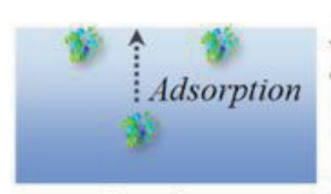

Gas phase
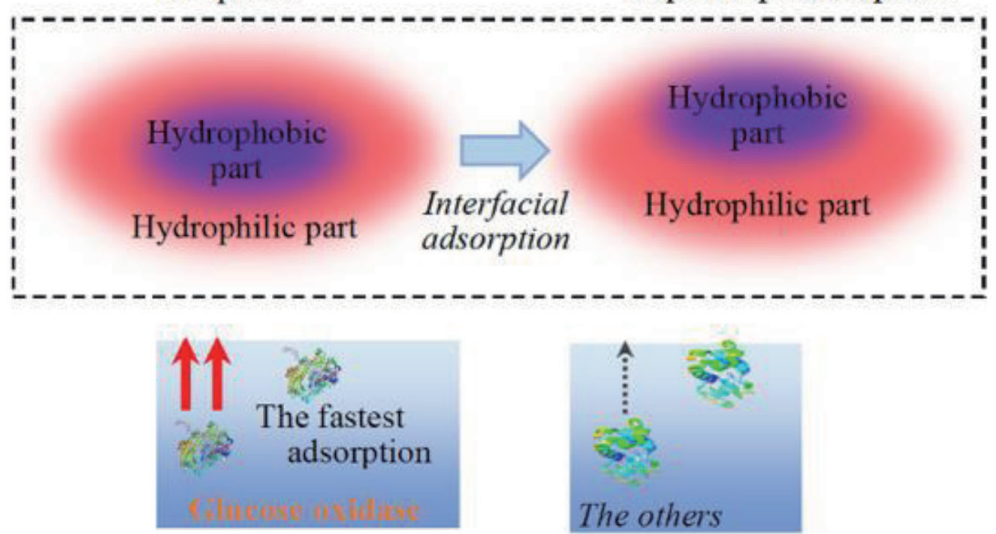
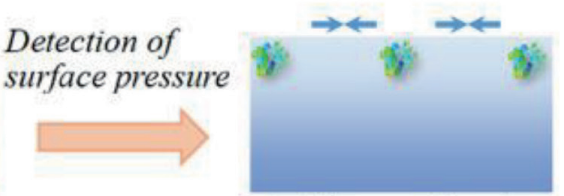

Liquid expansion phase

Fig. 3 (a) $\pi$-t isotherm of Gibbs monolayer at the water surface of glucose oxidase $\left(15^{\circ} \mathrm{C}\right)$. (b) Comparison of $\pi$-t isotherms of Gibbs monolayers on the water surface of glucose oxidase with other biomolecules $\left(15^{\circ} \mathrm{C}\right)$. (c) Schematic illustration of interfacial adsorption behavior and liquid expanded film transition.

phase to the liquid expansion phase. Before this phenomenon, the transition of the biomolecule from the water-soluble conformation to the amphiphilic conformation proceeds immediately after subphase preparation/static standing. Even if adsorption of biomolecules transformed into an amphiphilic conformation occurs at the air/water interface, it remains in a two-dimensional gas phase until interactions between hydrophobic chains occurs. Trypsin, luciferase, and glucose oxidase rapidly undergo interfacial conformational transitions, air/water interface adsorption, and the 

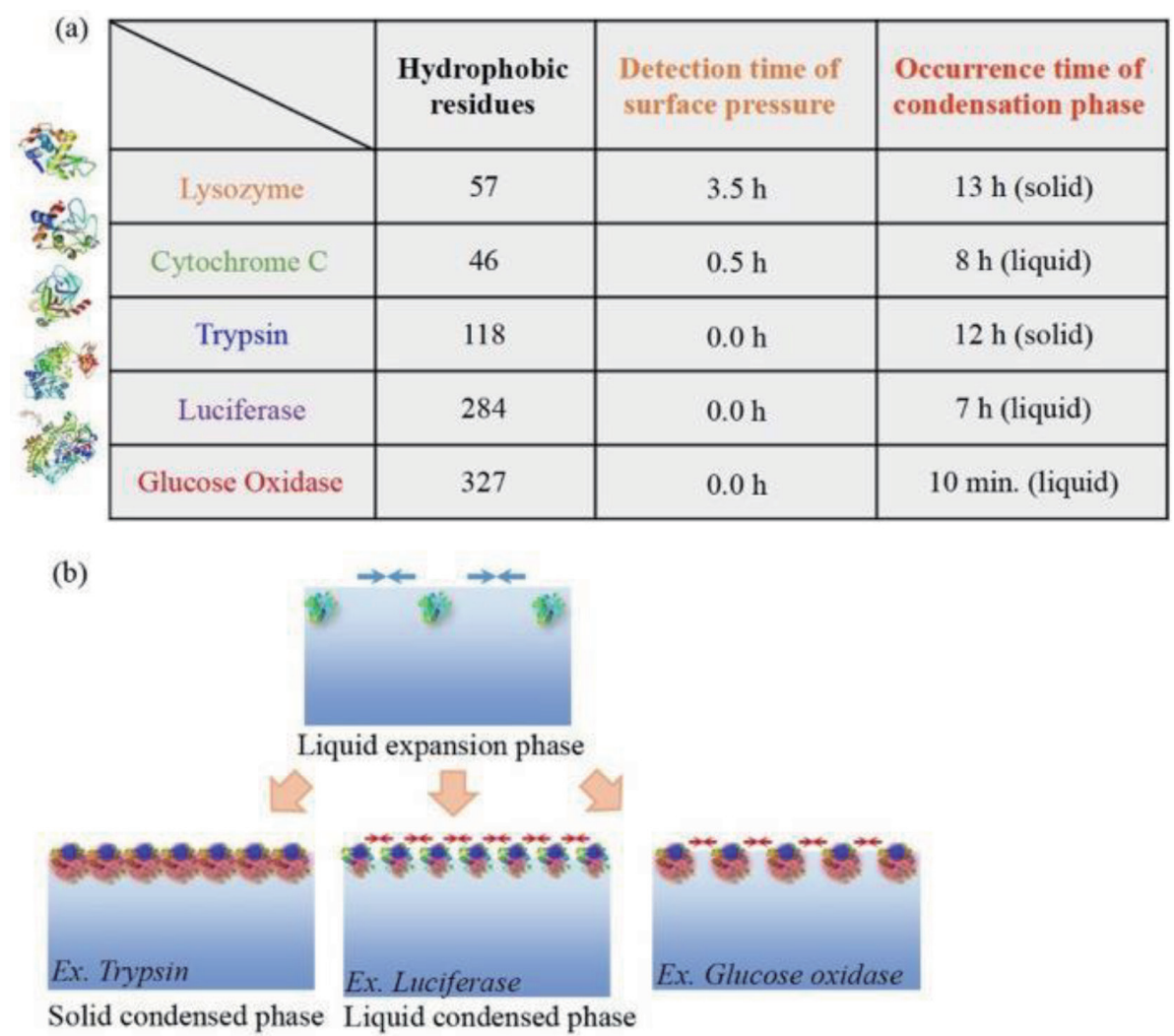

Fig. 4 (a) List of apparent hydrophobic residues of each biomolecule and transition parameters in Gibbs monolayer. (b) Schematic illustration of transition from liquid expansion film to each condensed film.

occurrence of interactions between hydrophobic groups. When discussing the stability of the water-soluble conformation, the stability is higher in the order of lysozyme, then cytochrome C. Our next focus was the transition from the liquid expansion phase to the condensed phase. The transition to the condensed phase increased the stability of the monolayer, but was equivalent to that of the deactivated state as a biomolecule because of its high density. In addition, due to the steepness of transitions in the isotherms, the transition to the solid condensed film and the transition to the liquid condensed film could be differentiated. Lysozyme and trypsin transferred to the solid condensed phase required $13 \mathrm{~h}$ and $12 \mathrm{~h}$, respectively. The persistence time of the liquid expansion phase was long, and the stability of the interfacial adsorption denaturation state before deactivation was high. On the other hand, cytochrome C, luciferase, and glucose oxidase required 8,7 , and $3 \mathrm{~h}$, respectively, before transfer to the liquid condensation membrane. The time that glucose oxidase remained in the liquid expansion phase was particularly short. It is likely that the number of hydrophobic residues, such as valine and isoleucine, in each biomolecule will have a strong influence on this tendency. Lysozyme and cytochrome C have approximately 57 and 46 hydrophobic residues, respectively. The number of hydrophobic residues in trypsin is more than double, and the two-dimensional gas phase could not be confirmed, but it required half a day for the condensed phase transition. Luciferase and glucose oxidase, which have two to three times more hydrophobic residues, have no gas phase, and display rapid transition to the liquid expansion phase. Comparing the saturated surface pressure, lysozyme, glucose oxidase, cytochrome C, trypsin, and luciferase changed in this order from low to high values. Cytochrome $\mathrm{C}$ and trypsin reversed their saturated surface pressure values over time, that is, after the condensed phase transition of cytochrome C. The saturated surface pressure value is expected to reflect the relative film density. Glucose oxidase has a large number of hydrophobic residues, and the rapid increase in van der Waals forces between hydrophobic groups produced a steep rise in surface pressure. However, as far as we can guess from the data, the monolayer density itself is low even after the condensed phase transition, and the hydrophilic groups are considered to protrude below the water surface in wide space $^{38,39)}$. Therefore, it is expected that the degree of freedom between hydrophilic groups would be maintained. On the other hand, since luciferase was packed most densely in the Gibbs monolayer, even in the liquid-expanded state, it can be predicted that luciferase would exhibit a high specific saturation surface pressure value. The above contents are summarized in Fig. 4(b).

Figure 5 illustrates the morphological changes to the 


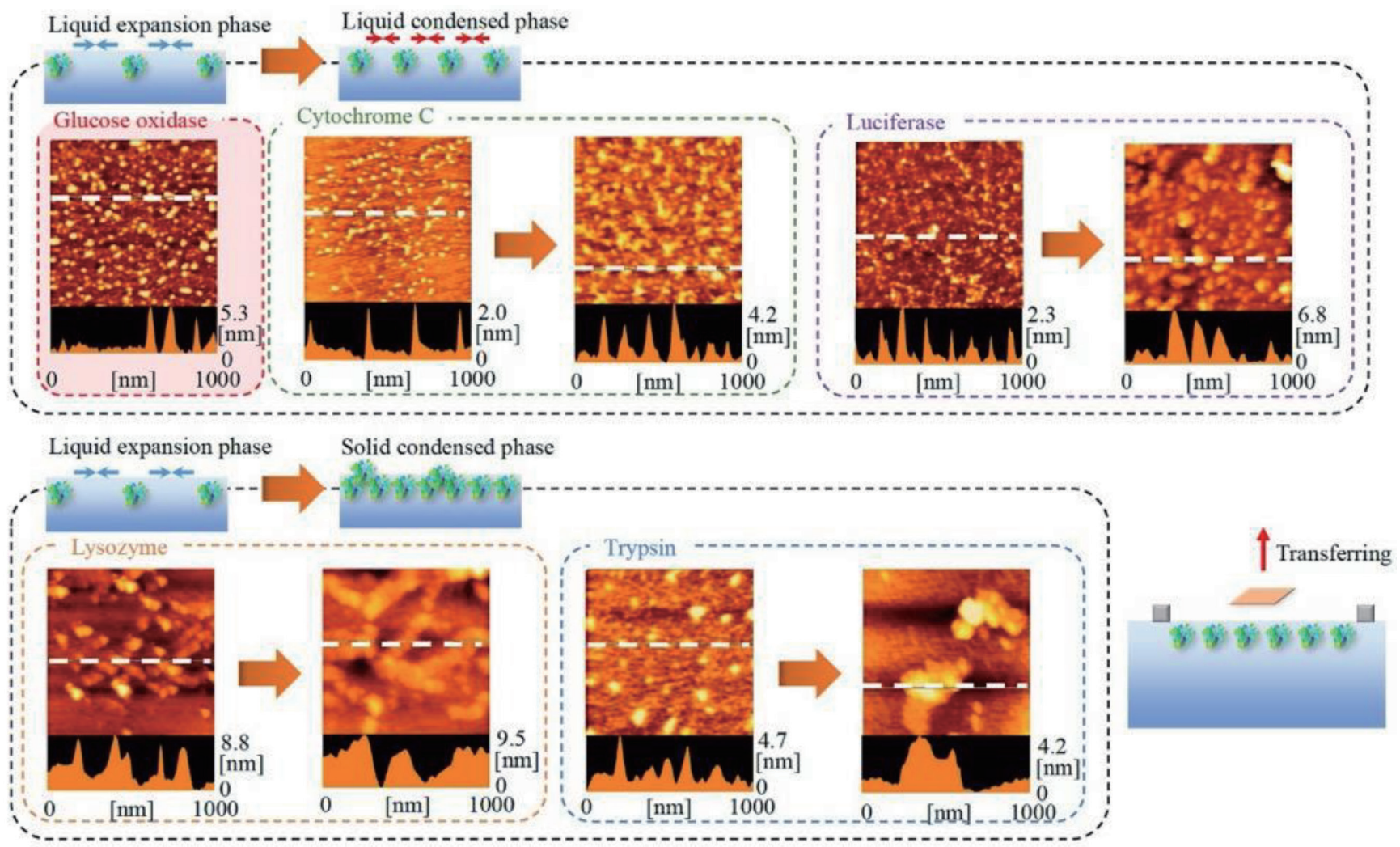

Fig. 5 AFM images of monolayers on solid surfaces for each biomolecule.

X-type single layer film transferred onto the solid substrate by the horizontal lifting method as an AFM image. On the left side of the upper column, the surface morphology of a monolayer of glucose oxidase in the condensed phase is shown. It is difficult to grasp the morphology of the glucose oxidase monolayer in the expanded film because the transition from the expanded phase to the condensed phase is completed in only approximately $10 \mathrm{~min}$. However, important information can also be obtained from the morphology of the condensed film. In the cytochrome $\mathrm{C}$ and luciferase monolayers that transferred to the liquid condensed phase, the size of the scattered domains in the condensed phase tended to be larger than that in the expanded phase. On the other hand, in the lysozyme and trypsin monolayers that transitioned to the solid condensed phase by the steep slope transition of the $\pi-\mathrm{t}$ curve, the domains tended to coalesce, and the boundaries tended to become diffuse. Glucose oxidase clearly followed the former tendency, and it is expected from the behavior of $\pi$-t that it is a biomolecule with the property of liquid condensed phase transition. Also, the distributions of domain size that can be read from AFM measurements are lysozyme: 130 to $150 \mathrm{~nm}$, trypsin: 80 to $100 \mathrm{~nm}$, cytochrome C: 30 to $40 \mathrm{~nm}$ (before transition)/ 60 to $70 \mathrm{~nm}$ (after transition), glucose oxidase: 60 to $70 \mathrm{~nm}$, and luciferase: 40 to $50 \mathrm{~nm}$ (before transition)/ 80 to $90 \mathrm{~nm}$ (after transition), respectively. On the other hand, as literature values, each molecular size is lysozyme:
$1.9 \mathrm{~nm} \times 3.2 \mathrm{~nm} \times 3.3 \mathrm{~nm}$, trypsin: $3.8 \mathrm{~nm} \times 3.8 \mathrm{~nm} \times 3.8$ $\mathrm{nm}$, cytochrome C: $2.6 \mathrm{~nm} \times 3.2 \mathrm{~nm} \times 3.3 \mathrm{~nm}$, and glucose oxidase: $7.0 . \mathrm{nm} \times 5.5 \mathrm{~nm} \times 8.0 \mathrm{~nm}$, have been given, respectively ${ }^{40)}$. A simple comparison of these biomaterials shows that the domain of Glucose oxidase is formed with a small aggregation size.

In this study, although the Gibbs adsorption monolayers are immobilized on a solid substrate by the horizontal Lifting method, the AFM images of the obtained LB films may not necessarily accurately reflect the state of the adsorption film at the air/water interface. Depending on the state of the adsorption film, good transfer like the Langmuir film is difficult. Especially in the horizontal lifting method, the substrate surface gets wet and pulls up during transfer, so even if enzyme molecules adhere to the substrate, molecules may be rearranged. It should be cared when evaluating the structure of the adsorption film from the AFM observation of the LB films. Essentially, if possible, it would be desirable to observe the film formation process directly on the water surface with a BAM or a fluorescence microscope.

\subsection{Consideration of interfacial adsorption behavior/ Gibbs monolayer formation of DNA molecules without conspicuous hydrophobic groups \\ In the conceptualization of "interfacial adsorption dena-} turation", the presence of apparent hydrophobic units in 
the relevant hydrophilic biomolecules, and their apparent total number, are important factors. Here, salmon testisderived DNA molecules had no apparent hydrophobic units. Therefore, the $\pi$-t isotherm of the Gibbs monolayer of this DNA molecule did not show a positive value, and exhibited a behavior that decreased over the error range
(Fig. 6(a)). However, do DNA molecules have no interfacial adsorption ability? In order to answer this question, a finite compression rate was applied to the Gibbs monolayer of DNA and surface pressure-area $(\pi-A)$ isotherms were measured. As a result, the retention time dependence was confirmed for the $\pi$-A curve, and detection of surface pres- (a)

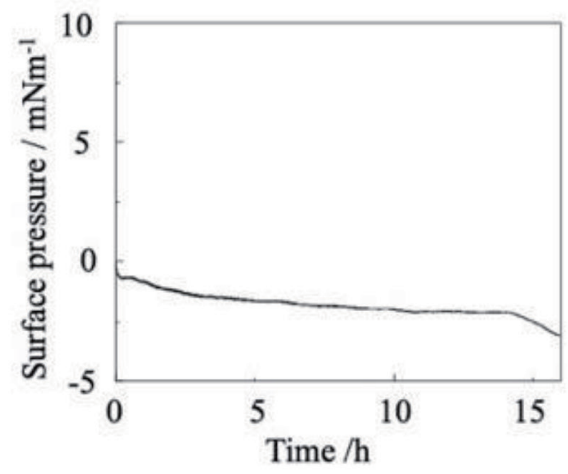

(c)
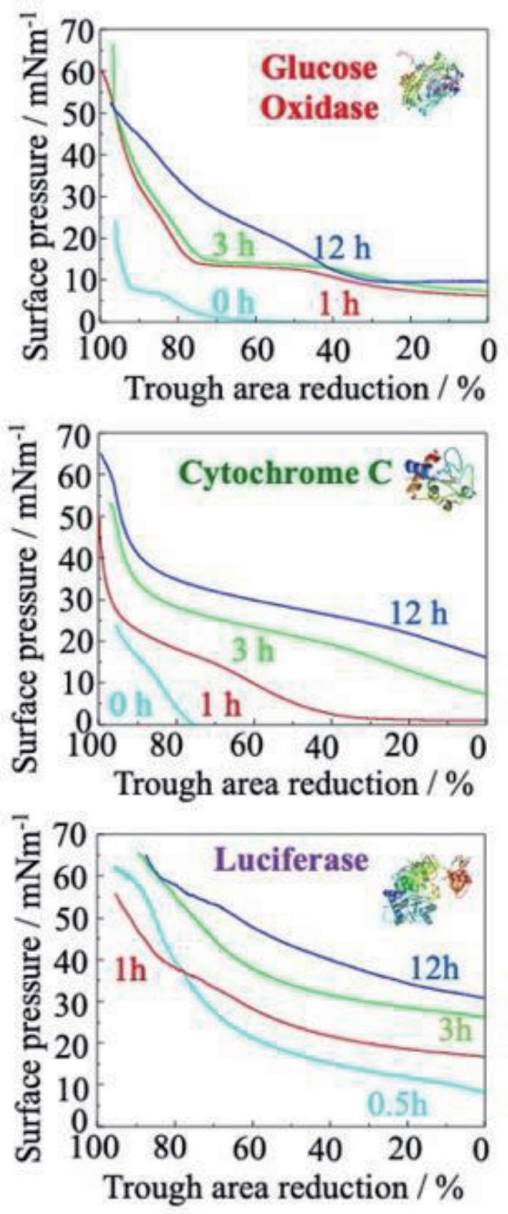

(b)
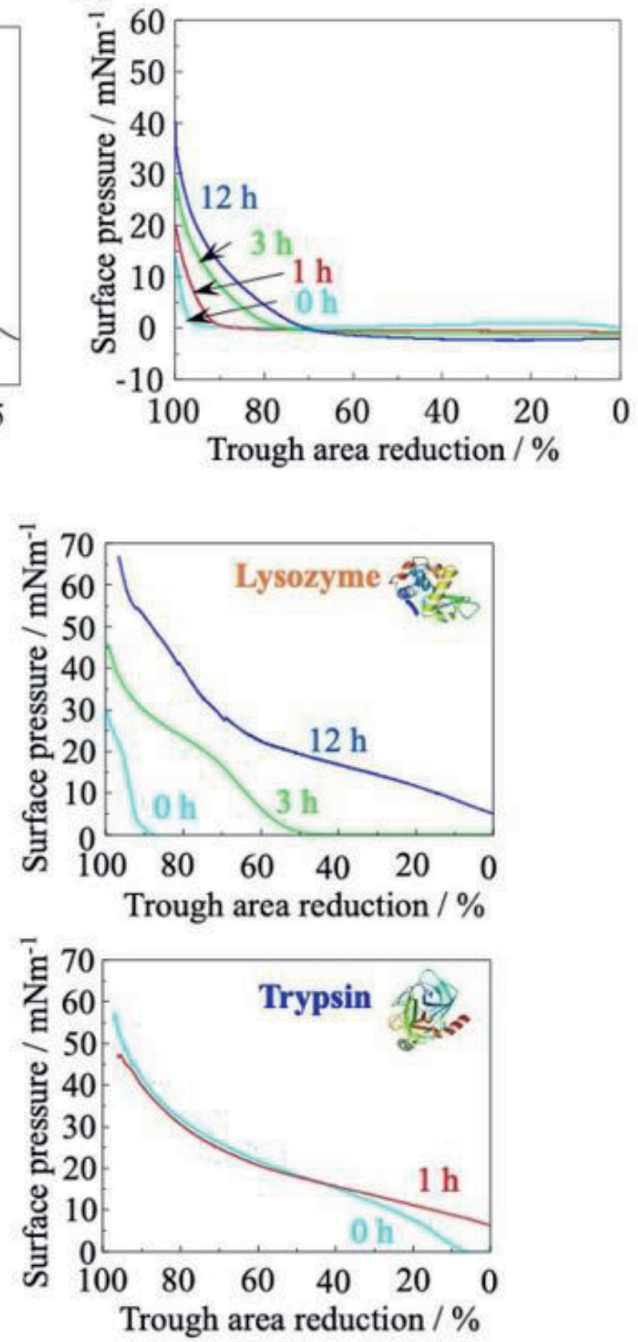

Fig. 6 (a) $\pi$-t isotherm of Gibbs monolayer on the water surface of salmon testis-derived DNA $\left(15^{\circ} \mathrm{C}\right)$. (b) $\pi$-A isotherms of Gibbs monolayer on the water surface of salmon testis-derived DNA $\left(15^{\circ} \mathrm{C}\right)$. (c) $\pi$-A isotherms of Gibbs monolayers on the water surface of each biomolecule $\left(15^{\circ} \mathrm{C}\right)$. In $(b)$ and $(c)$, the increase in initial surface pressure over time is not normalized. 
sure tended to become clear as the standing time increased (Fig. 6(b)). For reference, Fig. 6(c) shows the retention time dependence of the $\pi$-A isotherm for various biomolecules used in this study. All molecules clearly showed a $\pi$-A curve, and there were some molecular groups with a time dependence similar to that of DNA. As a result, it is expected that the DNA molecule used in this study can also form an interfacial adsorption monolayer. Therefore, in Fig. 7, film transfer onto the solid substrate was performed and evaluated in a state where the surface pressure was not detected together with the lysozyme enzyme. Lysozyme was transferred onto the substrate within $3 \mathrm{~h}$ after standing without the detection of surface pressure. The structure of the lysozyme aggregate and the domain-like structure of DNA were clearly confirmed in AFM images of these monolayers. In addition, the presence of alkyl groups in DNA and the presence of amide groups in lysozyme could be confirmed from the results of IR measurements involving both multilayers. In other words, the conclusions drawn from these results are that the interfacial adsorption of biomolecules progresses even in the two-dimensional gas phase state where surface pressure is not detected, and at the same time, a conformational transition to the amphipathic one also occurs. The basis for this reasoning is the existence of studies in which similar studies have been made on lysozyme and trypsin enzymes in the past, and conformational changes in hydrophilic-hydrophobic groups were supported during the formation of air/water interfacial adsorption monolayers ${ }^{41,42)}$. In this study, it is supported the logic of conformational changes during the forma-

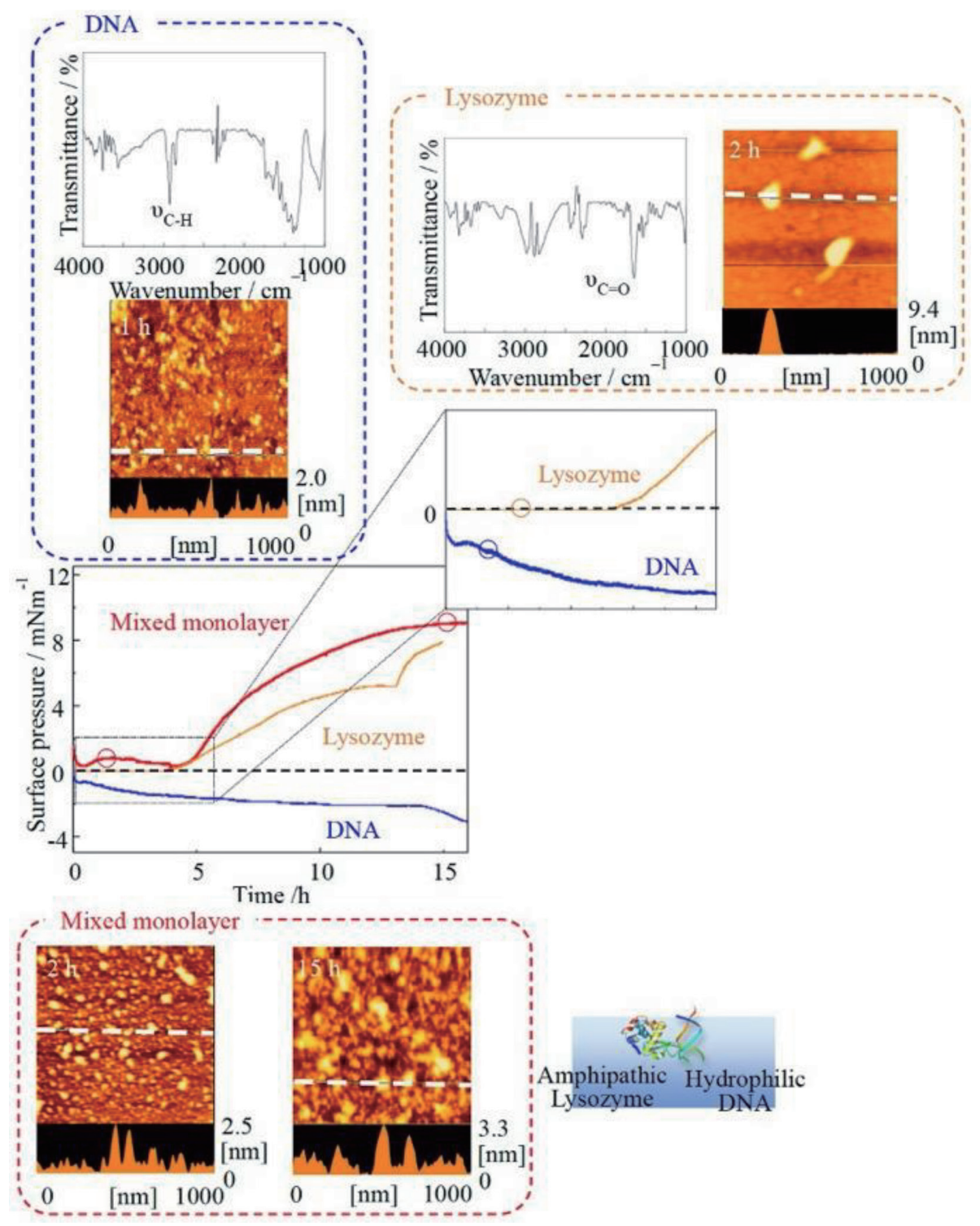

Fig. 7 Surface morphology of mixed monolayers of salmon testis-derived DNA and lysozyme at a given surface pressure, IR spectra of each multilayer, and the $\pi$-t curve and corresponding AFM images of such monolayers. 
tion of adsorption membranes of other biomolecules according to these study examples.

By the way, a salmon testis-derived DNA shows a reproducible decrease in surface pressure over time. It seems that this tendency would not be an experimental error. On the other hand, when measuring the $\pi$-A curve of salmon testis-derived DNA, surface pressure is detected as compression progresses, and it is expected that this biomolecule has the ability to form a monolayer on the water surface. In addition, the fact that a biomolecule exists on the substrate can be understood from IR and AFM through the process of film transfer from the water surface in the state where the surface pressure of the $\pi$-t curve is zero. In other words, although the salmon testis-derived DNA is not dense enough to detect positive surface pressure, it can be concluded that a monolayer was present near the interface from a very early time point. However, since there is no clear hydrophobic group in the chemical structure of salmon testis-derived DNA, it is expected that the adsorbed amount of DNA that was sparsely present in the two-dimensional gas phase near the interface decreased over time. There are several reports of amphipathic performance and lack of hydrophobicity of these DNA molecules $^{43,44)}$. Therefore, the low hydrophobicity of these DNAs is corresponding to the evidence of the above reasoning.

In the case where surface pressure is not detected, such as in DNA or the initial state of lysozyme, although lateral intermolecular interactions will not be detected, it is expected that biomolecular functions may not be deactivated. However, it may be unlikely that the DNA used in this study formed a stable monolayer. Therefore, we investigated the stabilization of DNA molecules as a monolayer. For this purpose, the mixed monolayer technique was used. For example, in the case of a dye molecule having a bulky chromophore, stabilization can be promoted by the formation of a binary mixed monolayer with long-chain fatty acids and/or ternary mixed monolayers with long-chain fatty acids and $n$-alkanes ${ }^{45)}$. Therefore, this presents a means to form a stable monolayer of DNA in a mixed film by forming a binary monolayer of DNA and lysozyme. When a mixed film of DNA and lysozyme is formed at a molar ratio of $1: 1$, the $\pi$-t curve showed a positive surface pressure value from the initial state, and the surface pressure value increased remarkably after $5 \mathrm{~h}$. At this time, the steep two-dimensional transition showing a solid condensed phase transition peculiar to lysozyme disappeared, and the surface morphology also changed to the domain size-increasing behavior. That is to say, it can be understood that the DNA monolayer was incorporated into the lysozyme monolayer, and these interacted with each other to change the $\pi$-t curve, which affected the behavior of the two-dimensional transition. Since the change in the $\pi$-t curve indicates the existence of an interaction between the two molecules, there is concern that the functions of both molecules may be deactivated. However, on the other

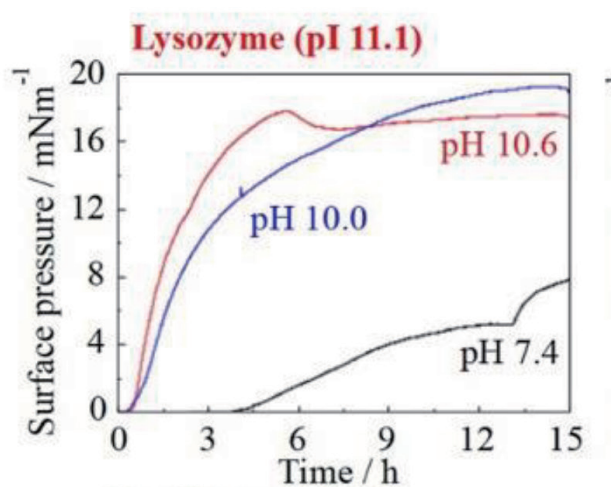

\section{Cytochrome C (pI 10.7)}

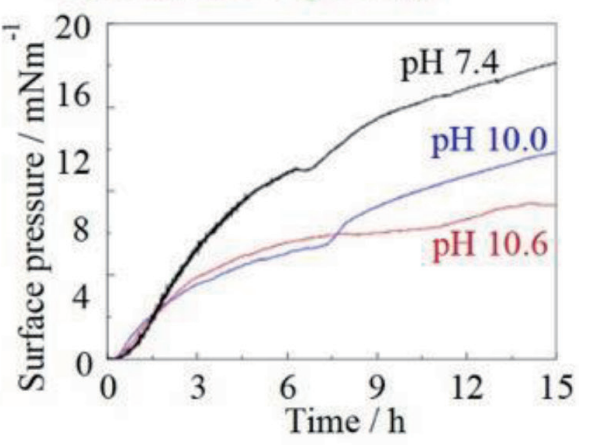

Luciferase (pI 8.1)

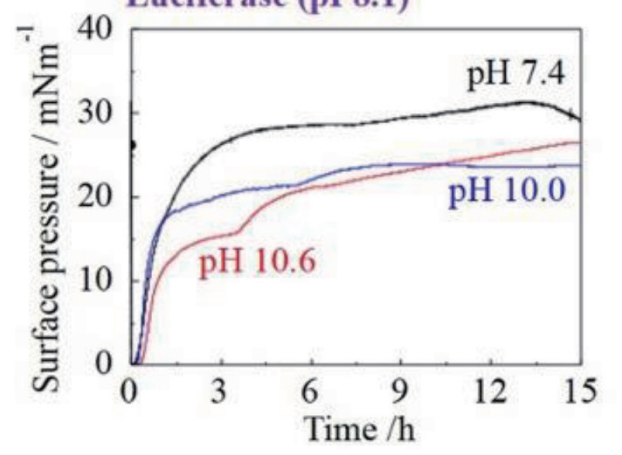

Fig. $8 \mathrm{pH}$ dependence of $\pi$-t isotherm of Gibbs monolayer for each biomolecule $\left(15^{\circ} \mathrm{C}\right)$. 
hand, it can be understood that both can coexist in a stable monolayer state in the low surface pressure region without showing phase separation or affinity. It can be expected that such a method of employing mixed monolayers will effectively contribute to the selective functional utilization of interfacial adsorption denaturation molecules.

\subsection{Other factors affecting interfacial adsorption denatur- ation and extension of the corresponding theory}

To date, the interfacial adsorption denaturation behavior of biomolecules has been investigated from the viewpoint of Gibbs monolayer formation, focusing on glucose oxidase and salmon testis-derived DNA molecules. However, this study has been performed using a buffer solution containing biomolecules at $\mathrm{pH} 7.4$ under $15^{\circ} \mathrm{C}$ lower phase water conditions. In general, when the temperature of the subphase rises, the monolayer expands and shows a curve of gradual surface pressure change. Therefore, such experiments should be conducted under the condition that the subphase temperature is kept constant. Furthermore, most of the biomolecules used in this study have isoelectric points on the basic side. Here, the subphase temperature was kept constant at $15^{\circ} \mathrm{C}$, the $\mathrm{pH}$ of the buffer solution was adjusted, and changes in the $\pi$-t curve were confirmed (Fig. 8). The $\pi$-t curves of lysozyme, cytochrome C, and luciferase changed significantly after $15 \mathrm{~h}$ of measurement. Generally, it is known that the stability of biomolecules as a monolayer film improves as the basicity increases and the isoelectric point approaches ${ }^{46)}$. Specifically, there are reports that biomolecules near the isoelectric point become more hydrophobic and the role of hydrophobic groups is clarified, which enhances the stability of the adsorbed monolayer ${ }^{47,48)}$. The existence of these reports is the basis for the above reasoning. From the above, it was found that $\mathrm{pH}$ dependence, considering the isoelectric point, can be a factor affecting interfacial adsorption behavior.

The above results are summarized in Fig. 9. In a previous report $^{11,12)}$, the interfacial adsorption denaturation of lyso-

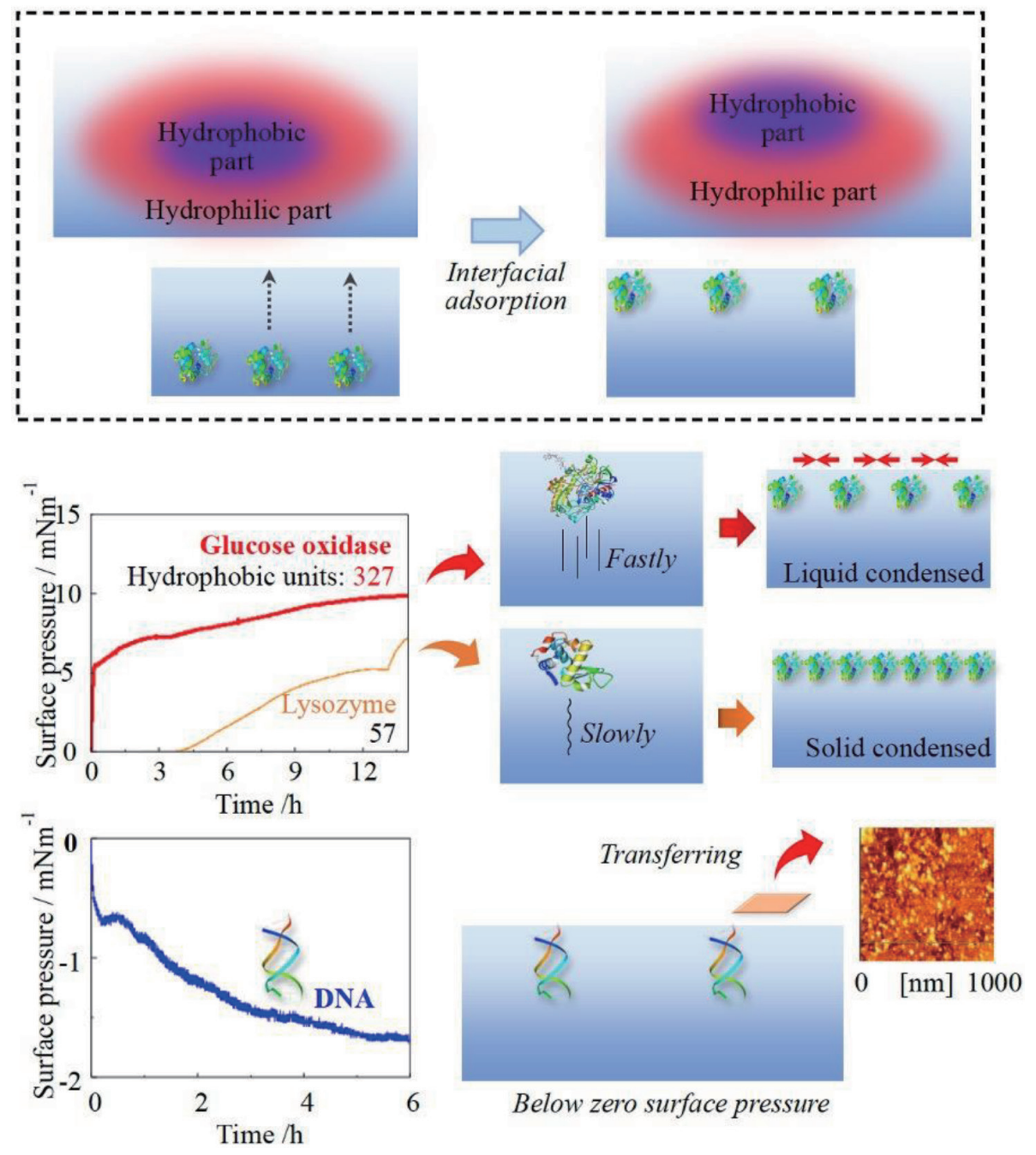

Fig. 9 Summarized results in this study. 
zyme, trypsin, cytochrome $\mathrm{C}$, and luciferase was proposed through Gibbs monolayer formation. Glucose oxidase exhibited a faster increase in surface pressure than any of the other enzymes/proteins, with a rapid transition to the amphipathic conformation. It is expected that this is because the apparent number of hydrophobic residues in glucose oxidase was overwhelmingly high. However, the absolute value of the saturated surface pressure detected did not reach that of luciferase, trypsin, and cytochrome $\mathrm{C}$, and no solid condensed phase appeared. This is an example of where the rate of transition from the two-dimensional gas to liquid expansion phases is not proportional to the density or stability of the Gibbs monolayer formed. In addition, the domain-like morphology supports the fact that no solid condensed phase was exhibited. In addition, it was found that interfacial adsorption and conformational transition occurred, even in the initial state of lysozyme, and the stage of zero surface pressure of salmon testis-derived DNA. Stabilization as a Gibbs monolayer is thought to be promoted by the formation of a mixed film with a more stable monolayer-forming substance. It was also found that such an interfacial adsorption transition can also affect increased hydrophobic status caused by $\mathrm{pH}$ dependence of each biomolecule with regard to the isoelectric point. As mentioned previously, it will be extremely important to understand that concentration and $\mathrm{pH}$ also affect dynamic surface tension and interfacial denaturation.

In order to maintain the functions of a biomolecule, develop new functions, or enhance existing functions, it would be interesting to establish a special state by simply controlling the transition behavior of a well-known biomolecule. There is also the ability to further investigate by monitoring the behavior of known substances, rather than expending the effort required to create or explore new substances. In the future, there will be efforts seeking functionality that would allow each biomolecule to be expressed during such interface adsorption denaturation phenomena.

\section{Conclusion}

This study was conducted to investigate the conformational changes involving biomolecules and air/water interfacial adsorption, termed "interfacial adsorption denaturation", and the subsequent organizational state change in the same plane. Specifically, we used glucose oxidase and DNA from salmon testes to attempt to extend this idea of interfacial adsorption denaturation. The $\pi$-t isotherm of the glucose oxidase Gibbs monolayer showed a rapid increase in surface pressure and a relatively rapid transition to the liquid condensed film. The appearance of this rapid liquid expansion phase occurred much earlier than the enzymebased molecular groups compared in this study. The results of this experiment were related to the number of hydrophobic residues in the building blocks, with the number of hydrophobic residues in glucose oxidase being the highest of these biomolecules. This result is consistent with the proposal in previous reports ${ }^{11,12}$. On the other hand, the DNA used in this study did not have a unit referred to as a hydrophobic group, and did not present a positive surface on the $\pi$-t curve. However, in the air/water interface field, interfacial adsorption occurred at zero surface pressure, and the existence of molecules at the air/water interface was confirmed, even in such a two-dimensional gas phase state. Furthermore, it was found that the increase in surface pressure and the change from the behavior of each single component molecule were detected by the formation of a mixed film of DNA and biomolecules forming a stable Gibbs monolayer. Furthermore, when $\mathrm{pH}$ dependence was evaluated by considering the isoelectric points of the biomolecule groups, the interfacial adsorption denaturation behavior changed. This was due to increased stabilization of the hydrophobicity of the biomolecules, and it was found that the increased hydrophobicity of the molecules greatly affected interfacial adsorption denaturation.

\section{Acknowledgment}

This study was supported by JSPS KAKENHI Scientific Research on Innovative Areas "MSF Materials Science" (Grant Number JP 19H05118).

\section{References}

1) Guan, W.; Ni, Z.; Hu, Y.; Liang, W.; Ou, C. et al. Clinical characteristics of 2019 novel coronavirus infection in China. N. Engl. J. Med. 382, 1708-1720 (2020).

2) Zhou, F.; Yu, T.; Du, R.H.; Fan, G.H.; Liu, Y. et al. Clinical course and risk factors for mortality of adult inpatients with COVID-19 in Wuhan, China: a retrospective cohort study. Lancet 395, 1054-1062 (2020).

3) Anderson, R.M.; May, R.M. Vaccination and herd immunity to infectious diseases. Nature 318, 323-329 (1985).

4) Larson, H.J. Vaccine trust and the limits of information. Science 353, 1207-1208(2016).

5) Burch, C.L.; Chao, L. Evolvability of an RNA virus is determined by its mutational neighbourhood. Nature 406, 625-628(2000).

6) Esvelt, K.M.; Carlson, J.C.; Liu, D.R. A system for the continuous directed evolution of biomolecules. Nature 472, 499-U550 (2011).

7) Bellissent-Funel, M.C.; Hassanali, A.; Havenith, M.; Henchman, R.; Pohl, P. et al. Water Determines the Structure and Dynamics of Proteins. Chem. Rev. 116, 
7673-7697(2016).

8) Minasyan, H. Sepsis and septic shock: Pathogenesis and treatment perspectives. J. Crit. Care 40, 229-242 (2017).

9) Park, J.Y.; Park, S.; Choe, W.; Yong, H.I.; Jo, C. et al. Plasma-Functionalized Solution: A Potent Antimicrobial Agent for Biomedical Applications from Antibacterial Therapeutics to Biomaterial Surface Engineering. ACS Appl. Mater. Interfaces 9, 43470-43477 (2017).

10) Ibrahim, H.R.; Higashiguchi, S.; Juneja, S.R.; Kim, M.; Yamamoto, T. A structural phase of heat-denatured lysozyme with novel antimicrobial action. J. Agric. Food Chem. 44, 1416-1423(1996).

11) Yunoki, T.; Kimura, Y.; Fujimori, A. Division of roles of modified chains in rgano-magnetic nanoparticles using Organo-modified agents having hydrophilic reactive polar groups at both ends.: Formation of high-density single-particle layers and bioconjugation. Colloids Surf. B 173, 759-768(2019).

12) Yunoki, T.; Kimura, Y.; Fujimori, A. Maintenance Properties of Enzyme Molecule Stereostructure at High Temperature by Adsorption on Organo-Modified Magnetic Nanoparticle Layer Template. Bull. Chem. Soc. Jpn. 92, 1662-1671 (2019).

13) Blodgett, K. Monolayer films of fatty acids on glass. $J$. Am. Chem. Soc. 56, 495 (1934).

14) Petty, M.C. Langmuir-Blodgett films. Cambridge Univ. Press, New York (1996).

15) Ulman, A. An Introduction to Ultrathin Organic Films, from Langmuir-Blodgett to Self-assembly. Academic Press, Boston (1991).

16) Vollhardt, D.; Melzer, V. Phase transition in adsorption layers at the air-water interface: Bridging to Langmuir monolayers. J. Phys. Chem. B 101, 3370-3375 (1997).

17) Gaines, Jr., G.L. Insoluble monolayers at liquid gas interfaces. Wiley, New York (1966).

18) Wang, Z.D.; Gurel, O.; Baatz, J.E.; Notter, R.H. Acylation of pulmonary surfactant protein-C is required for its optimal surface active interactions with phospholipids. J. Biol. Chem. 271, 19104-19109 (1996).

19) Rapaport, H.; Kuzmenko, I.; Berfeld, M.; Kjaer, K.; AlsNielsen, J. et al. From nucleation to engineering of crystalline architectures at air-liquid interfaces. $J$. Phys. Chem. B 104, 1399-1428(2000).

20) Kovalenko, A.; Polavarapu, P.; Pourroy, G.; Waton, G.; Krafft, M.P. pH-Controlled Microbubble Shell Formation and Stabilization. Langmuir 30, 6339-6347 (2014).

21) Kinoshita, K.; Parra, E.; Needham, D. New sensitive micro-measurements of dynamic surface tension and diffusion coefficients: Validated and tested for the adsorption of 1-Octanol at a microscopic air-water interface and its dissolution into water. J. Colloid Interface Sci. 488, 166-179(2017).
22) Kraack, H.; Ocko, B.M.; Pershan, P.S.; Sloutskin, E.; Deutsch, M. Structure of a Langmuir film on a liquid metal surface. Science 298, 1404-1407 (2002).

23) Matshaya, T.J.; Lanterna, A.E.; Granados, A.M.; Krause, R.W.M.; Maggio, B. et al. Distinctive Interactions of Oleic Acid Covered Magnetic Nanoparticles with Saturated and Unsaturated Phospholipids in Langmuir Monolayers. Langmuir 30, 5888-5896 (2014).

24) Mottola, M.; Vico, R.V.; Villanueva, M.E.; Fanani, M.L. Alkyl esters of L-ascorbic acid: Stability, surface behaviour and interaction with phospholipid monolayers. J. Colloid Interface Sci. 457, 232-242 (2015).

25) Peikert, M.; Chen, X.D.; Chi, L.F.; Brezesinski, G.; Janich, S. et al. Phase Behavior and Molecular Packing of Octadecyl Phenols and their Methyl Ethers at the Air/Water Interface. Langmuir 30, 5780-5789 (2014).

26) Duarte, F.; Paton, R.S. Molecular Recognition in Asymmetric Counteranion Catalysis: Understanding Chiral Phosphate-Mediated Desymmetrization. J. Am. Chem. Soc. 139, 8886-8896 (2017).

27) Ekelund, K.; Sparr, E.; Engblom, J.; Wennerstrom, H.; Engstrom, S. An AFM study of lipid monolayers. 1. Pressure-induced phase behavior of single and mixed fatty acids. Langmuir 15, 6946-6949 (1999).

28) Naolou, T.; Hussain, H.; Baleed, S.; Busse, K.; Lechner, B.D. et al. The behavior of fatty acid modified poly (glycerol adipate) at the air/water interface. Colloids Surf. A 468, 22-30 (2015).

29) Micklitsch, C.M.; Medina, S.H.; Yucel, T.; Nagy-Smith, K.J.; Pochan, D.J. et al. Influence of Hydrophobic Face Amino Acids on the Hydrogelation of beta-Hairpin Peptide Amphiphiles. Macromolecules 48, 1281-1288 (2015).

30) Eskici, G.; Axelsen, P.H. Amyloid Beta Peptide Folding in Reverse Micelles. J. Am. Chem. Soc. 139, 95669575(2017).

31) Wohlfahrt, G.; Witt, S.; Hendel, J.; Schomburg, D.; Kalisz, H.M. et al. 1.8 and 1.9 angstrom resolution structures of the Penicillium amagasakiense and Aspergillus niger glucose oxidases as a basis for modelling substrate complexes. Acta Crystallogr, Sect. D: Biol. Crystallogr. 55, 969-977 (1999).

32) Mazzoli, A.; Spalletti, A.; Carlotti, B.; Emiliani, C.; Fortuna, C.G. et al. Spectroscopic Investigation of Interactions of New Potential Anticancer Drugs with DNA and Non-Ionic Micelles. J. Phys. Chem. B 119, 1483$1495(2015)$.

33) Sun, H.F.; Li, S.K.; Qi, W.; Xing, R.R.; Zou, Q.L. et al. Stimuli-responsive nanoparticles based on co-assembly of naturally-occurring biomacromolecules for in vitro photodynamic therapy. Colloids Surf. A 538, 795-801 (2018).

34) Saranya, S.; Justin, S.J.S.; Solomon, R.V.; Wilson, P. L- 


\section{Y. Kimura, Y. Mashiyama, H. Maruyama et al.}

arginine directed and ultrasonically aided growth of nanocrystalline hydroxyapatite particles with tunable morphology. Colloids Surf. A 538, 270-279 (2018).

35) Fukuda, K.; Nakahara, H.; Kato, T. Monolayers and multilayers of anthraquinone derivatives containing long alkyl chains. J. Colloid Interf. Sci. 54, 430-438 (1976).

36) Zaitsev, S.Y. Functional Langmuir films with glucose oxidase. Colloids Surf. A 75, 211-216(1993).

37) Rinuy, J.; Brevet, P.F.; Girault, H.H. Second harmonic generation of glucose oxidase at the air/water interface. Biophys. J. 77, 3350-3355 (1999).

38) Rosilio, V.; Boissonnade, M.M.; Zhang, J.Y.; Jiang, L.; Baszkin, A. Penetration of glucose oxidase into organized phospholipid monolayers spread at the solution/ air interface. Langmuir 13, 4669-4675 (1997).

39) Lee, Y.L.; Lin, J.Y.; Lee, S. Adsorption behavior of glucose oxidase on a dipalmitoylphosphatic acid monolayer and the characteristics of the mixed monolayer at air/liquid interfaces. Langmuir 23, 2042-2051 (2007).

40) Hartmann, M. Ordered mesoporous materials for bioadsorption and biocatalysis. Chem. Mater. 17, 45774593(2005).

41) Miao, S.D.; Leeman, H.; De Feyter, S.; Schoonheydt, R.A. Three-Component Langmuir-Blodgett Films Consisting of Surfactant, Clay Mineral, and Lysozyme: Construction and Characterization. Chem. Eur. J. 16,
2461-2469 (2010).

42) Saha, S.; Chowdhury, J. Sustained and improved enzymatic activity of trypsin immobilized in the Langmuir Blodgett film of DPPC: A rapid enzyme sensor for the detection of Azocasein. Mater. Chem. Phys. 243, 122647 (2020).

43) Lyadinskaya, V.V.; Lin, S.Y.; Michailov, A.V.; Povolotskiy, A.V.; Noskov, B.A. Phase Transitions in DNA/Surfactant Adsorption Layers. Langmuir 32, 1343513445 (2016).

44) Kang, X.L.; Chen, Q.B.; Shang, Y.Z.; Liu, H.L. Interaction between Gemini and DNA at the Air/Water Interface. Acta Phys. -Chim. Sin. 27, 1467-1473(2011).

45) Ray, K.; Nakahara, H. Spectroscopic and structural studies of cyanine dyes in functionalized LangmuirBlodgett films: electron and energy transfer processes. Jpn. J. Appl. Phys. 40, 5095-5104(2001).

46) Gorg, A.; Weiss, W.; Dunn, M.J. Current two-dimensional electrophoresis technology for proteomics. Proteomic 4, 3665-3685 (2004).

47) Hristova, S.H.; Zhivkov, A.M. Electrooptical determination of the isoelectric point of globular proteins: Cytochrome c adsorbed on montmorillonite nanoplates. Colloids Surf. B 176, 480-487 (2019).

48) Hristova, S.H.; Zhivkov, A.M. Isoelectric point of free and adsorbed cytochrome c determined by various methods. Colloids Surf. B 174, 87-94(2019). 